\title{
Riqueza de galhas de insetos em áreas de Caatinga com diferentes graus de antropização do estado da Bahia, Brasil
}

\author{
Gilda P. Brito' (D), Elaine C. Costa² (D), Sheila P. Carvalho-Fernandes ${ }^{3}$ (D) \& Juliana Santos-Silva ${ }^{4}$ (D)
}

\author{
1. Departamento de Ciências Humanas, Universidade do Estado da Bahia, Av. Contorno s/n, Centro, 46400-000 Caetité, BA, Brasil. \\ (gildaporto_ibce@hotmail.com). \\ 2. Departamento de Botânica, ICB, Universidade Federal de Minas Gerais, Av. Antônico Carlos, 31270-901, Belo Horizonte, MG, Brasil. \\ (elainecostabio@gmail.com). \\ 3. Departamento de Entomologia, Museu Nacional, Universidade Federal do Rio de Janeiro, Quinta da Boa Vista, São Cristóvão, $20940-040$ Rio de Janeiro, RJ, Brasil. \\ (sheilapcfernandes@gmail.com). \\ 4. Programa de Pós-graduação em Biodiversidade Vegetal, Departamento de Educação-DEDC, Universidade do Estado da Bahia, Rua da Gangorra, 503, CHESF \\ 48608-240, Paulo Afonso, BA, Brasil. (jussilva@uneb.br).
}

Recebido 27 de abril de 2017

Aceito 7 de fevereiro de 2018

Publicado 26 March 2018

DOI: $10.1590 / 1678-4766 e 2018003$

ABSTRACT. Richness of insect galls in Caatinga sites with different degrees of anthropogenic impact, Bahia State, Brazil. The current knowledge of insect galls in Caatinga vegetation is still only fragmentary, with most scientific efforts concentrated in only a few areas in the states of Pernambuco and Bahia. In the present study, we characterized morphologically insect galls in areas of Caatinga vegetation showing different degrees of anthropogenic impact in Ibiassucê, state of Bahia. Three sites were selected based on their levels of human disturbance, and four random plots (10 $\mathrm{m}^{2}$ each), separated from each other by $10 \mathrm{~m}$, were then established in each area, for a total of 12 plots. Twenty gall morphotypes were found on nine plant species belonging to eight families. The greatest gall diversity was found in sites showing intermediate levels of anthropogenic impacts ( $\mathrm{n}=8$ ), followed by heavily impacted areas $(n=7)$, and preserved areas $(n=5)$. Globoid galls, brown, with only a single chamber, glabrous, and separately disseminated were the most frequent. Lepidoptera, Coleoptera and Diptera (Cecidomyiidae) were the gall-forming insect orders. The results presented here show the importance of this kind of research and the need of inventories of galls in neglected Caatinga areas.

KEYWORDS. Bioindicators, gall-inducing insects, host plant, semiarid.

RESUMO. O conhecimento sobre galhas de insetos da Caatinga ainda é fragmentado, com grande parte do esforço científico concentrado em alguns poucos pontos nos estados de Pernambuco e Bahia. Este estudo teve como objetivo verificar a ocorrência de galhas entomógenas e realizar sua caracterização morfológica em áreas de Caatinga de Ibiassucê, Estado da Bahia, com diferentes intensidades de ação antrópica. Foram selecionadas três áreas com base nos seus diferentes graus de antropização. Em cada área amostrada foram estabelecidas quatro parcelas aleatórias de $10 \mathrm{~m}^{2}$ cada, com $10 \mathrm{~m}$ de distância entre si, perfazendo um total de 12 parcelas nos ambientes. Foram registrados 20 morfotipos de galhas distribuídos em nove espécies vegetais pertencentes a oito famílias. A maior riqueza de galhas foi encontrada nas áreas intermediárias ( $\mathrm{n}=8$ ), seguida das áreas antropizadas ( $\mathrm{n}=7$ ) e preservadas $(\mathrm{n}=5)$. Galhas globoides, marrons, com apenas uma câmara, glabras e disseminadas separadamente foram as mais frequentes. Lepidoptera, Coleoptera e Diptera (Cecidomyiidae) foram as ordens galhadoras. Os resultados aqui apresentados demonstram a importância desse tipo estudo e evidenciam a necessidade da realização de inventários de galhas em áreas pouco ou não amostradas da Caatinga.

PALAVRAS-CHAVE. Bioindicadores, insetos galhadores, plantas hospedeiras, semiárido.

A Caatinga, composta por um mosaico de florestas secas e vegetação arbustiva (savana-estépica), com enclaves de florestas úmidas montanas e de cerrados, é a única grande região natural brasileira cujos limites estão inteiramente restritos ao território nacional (TABARELLI \& SILVA, 2002).

A heterogeneidade ambiental da Caatinga e a singularidade de certos ambientes permitem supor a possibilidade de uma riqueza de insetos galhadores associada a essa vegetação. Entretanto, o aspecto que mais se destaca sobre as galhas neste bioma é o conhecimento insuficiente que se tem delas, com grande parte do esforço científico concentrado no estado de Pernambuco e recentemente na Bahia (SANTOS et al., 2011; CARVALHOFERNANDES et al., 2012).

O primeiro inventário de galhas realizado na Caatinga foi conduzido por SANTOS et al. (2011), onde foram estudadas áreas particulares dos municípios pernambucanos de Alagoinha, Custódia, Pombos, Bodocó, Serrita, Pesqueira, Salgueiro, Parnamirim, bem como do Parque Nacional do Vale do Catimbau e de dois parques estaduais de Pernambuco. Carvalho-Fernandes et al. (2012) inventariaram e caracterizaram as galhas de diferentes áreas de Caatinga próximas à Usina Hidroelétrica de Xingó pertencente à Companhia Hidroelétrica do São Francisco (CHESF). Os 
resultados desses estudos, além de evidenciarem a riqueza de galhas em ambientes de caatinga, reforçaram a necessidade de novos inventários de galhas em áreas ainda não estudadas desse ecossistema.

ACaatinga do centro-sul da Bahia - não diferentemente das outras regiões - também passa por um extenso processo de devastação ambiental. Suas áreas são alvos de exploração desordenada da vegetação natural através da extração de madeira, tanto para ser usada como lenha por comunidades da zona rural, quanto para servir de matéria-prima para pequenas indústrias da região (BrasiLeIro, 2009). Isto implica não só em perda de habitat como pode afetar todas as interações existentes, inclusive entre insetos e plantas (TABARELLI, 1998), em particular aquelas que originam a formação de galhas.

A fragmentação de habitat pode ter diferentes efeitos sobre as comunidades de insetos herbívoros. Em geral, a riqueza de insetos tende a diminuir com a redução da área de habitat disponível (e.g., Bolger et al., 2000; ALMEIDANeto et al., 2011). Além disso, os insetos mais especialistas podem ser substituídos por espécies generalistas, melhores adaptadas às perturbações antrópicas (e.g., HINEs et al., 2005; KAARTINEN \& RosLIN, 2011).

Os insetos galhadores respondem de maneira diferente aos diversos tipos de perturbação ambiental. JULĨ̃o et al. (2005) investigaram a influência da urbanização na riqueza e abundância de galhas em duas espécies vegetais e observaram que apenas a abundância era afetada, sendo nos ambientes com menor intensidade de ocupação encontrada um maior número de galhas. Por outro lado, outros estudos mostram que os insetos galhadores podem ser beneficiados pela mudança no habitat, mantendo populações estáveis ou até mesmo ter suas densidades aumentadas em áreas de borda em florestas secas (Altamirano et al., 2016) e em ambientes sob influência de atividades de mineração (ARAúso et al., 2014). Importantes ordens de insetos galhadores, como Diptera, Hymenoptera e Coleoptera, são mais diversas e abundantes em bordas do que no interior da vegetação (GoNZÁLEZ et al., 2017). A presença de vegetação com espécies de estágios iniciais e tardios nas bordas pode estar influenciando nesse efeito (González et al., 2015). Além disso, as comunidades de plantas que sofrem estresse ambiental são mais suscetíveis ao ataque de herbívoros (CHRISTIE et al., 2010). A alteração na abundância e riqueza de insetos galhadores em ambientes estressados demonstra o potencial bioindicador dessa guilda em ambientes antrópicos (JULIÃo et al., 2005; MoreIRA et al., 2007; AraúJo et al., 2012; ToMA et al., 2014).

Diante desse contexto, este estudo teve como objetivo investigar os efeitos das atividades antrópicas em comunidades de insetos galhadores em áreas de Caatinga de Ibiassucê (BA) com diferentes intensidades de ação humana.

\section{MATERIAL E MÉTODOS}

O estudo foi realizado em três áreas de Caatinga situadas no município de Ibiassucê, centro-sul da Bahia, Brasil. O clima no município é Semiárido de caráter atenuado, com pluviosidade de 700 a $800 \mathrm{~mm}$ anuais e temperatura média anual em torno de $21^{\circ} \mathrm{C}$ (IBGE, 2017). O relevo é ondulado ou suavemente ondulado, cujo tipo de solo predominante é o Latossolo Vermelho Amarelo (EMBRAPA SOLOS, 2006). Os tipos de vegetação predominantes são Caatinga e Floresta Estacional Decidual (CAR, 2007; MDA, 2010) e, menos de $0,6 \%$ da vegetação encontra-se protegida na forma de Área de Preservação Permanente e Reserva Legal de acordo com os dados do Censo Agropecuário 2006 (IBGE, 2006).

As áreas amostradas foram selecionadas com base na acessibilidade e nos seus diferentes estados de conservação (Tab. I; Figs 1-6). Baseado em uma escala de sucessão ecológica (CARVAlHo-Fernandes et al., 2012) e nos dados levantados sobre o uso prévio da terra obtidos através da consulta aos moradores antigos da região. Essas áreas foram classificadas como: (1) preservada - quando não houve exploração há pelo menos 20 anos; (2) intermediária - explorada há 10 anos e (3) antropizada - pressão antrópica constante, tais como pasto e cultivo de lavoura. Em cada área foram estabelecidas quatro parcelas aleatoriamente distribuídas de $10 \mathrm{~m}^{2}$ cada ( $2 \times 5$ metros de dimensões), com $10 \mathrm{~m}$ de distância entre si, perfazendo um total de 12 parcelas. Em cada uma dessas parcelas, as plantas foram vistoriadas à procura de galhas entre agosto de 2015 a julho de 2016, em diferentes períodos sazonais, sendo quatro excursões no período seco, duas no início (agosto e setembro) e duas no final (outubro e novembro), assim como no início (dezembro e fevereiro) e final da estação chuvosa (abril e julho).

As galhas, quando presentes, foram coletadas, fotografadas e as suas características morfológicas externas descritas (ex., órgão de ocorrência, cor, forma, pubescência e agrupamento). Para a terminologia do morfotipo de galha foi adotado o proposto por Isaias et al. (2013). O material coletado foi armazenado separadamente em sacos plásticos e transportado ao Laboratório de Botânica da Universidade do Estado da Bahia - DCH/Campus VI, Caetité para triagem.

Em laboratório, uma parte das galhas coletadas foi acondicionada em potes plásticos com papel toalha para a eclosão do inseto indutor. A outra parte foi dissecada com auxílio de uma lupa para retirada das formas imaturas, como também para observação do número de câmaras internas. As formas imaturas e indivíduos adultos dos insetos obtidos foram armazenados em álcool a 70\%. A identificação de algumas espécies de indutores foi realizada através de comparação dos

Tab. I. Coordenadas geográficas, altitude e estado de conservação das localidades amostradas no município de Ibiassucê, Bahia, Brasil.

\begin{tabular}{|c|c|c|c|}
\hline Localidade & $\begin{array}{l}\text { Coordenadas } \\
\text { geográficas }\end{array}$ & Altitude & $\begin{array}{c}\text { Estado de } \\
\text { Conservação }\end{array}$ \\
\hline Morro da Tapera & $\begin{array}{l}14^{\circ} 27^{\prime} 05^{\prime \prime} \mathrm{S} \\
42^{\circ} 15^{\prime} 17^{\prime \prime} \mathrm{W}\end{array}$ & $824 \mathrm{~m}$ & Preservado \\
\hline Quilombo Santo Inácio & $\begin{array}{c}14^{\circ} 22^{\prime} 0.1 \text { ' } \mathrm{S} \\
42^{\circ} 15^{\prime} 17.4 \text { ' } \mathrm{W}\end{array}$ & $585 \mathrm{~m}$ & Intermediário \\
\hline $\begin{array}{l}\text { Fazenda Tanque da } \\
\text { Mandioca }\end{array}$ & $\begin{array}{l}14^{\circ} 22^{\prime} 14.3^{\prime \prime} \mathrm{S} \\
42^{\circ} 15^{\prime} 13.5^{\prime \prime} \mathrm{W}\end{array}$ & $565 \mathrm{~m}$ & Antropizado \\
\hline
\end{tabular}



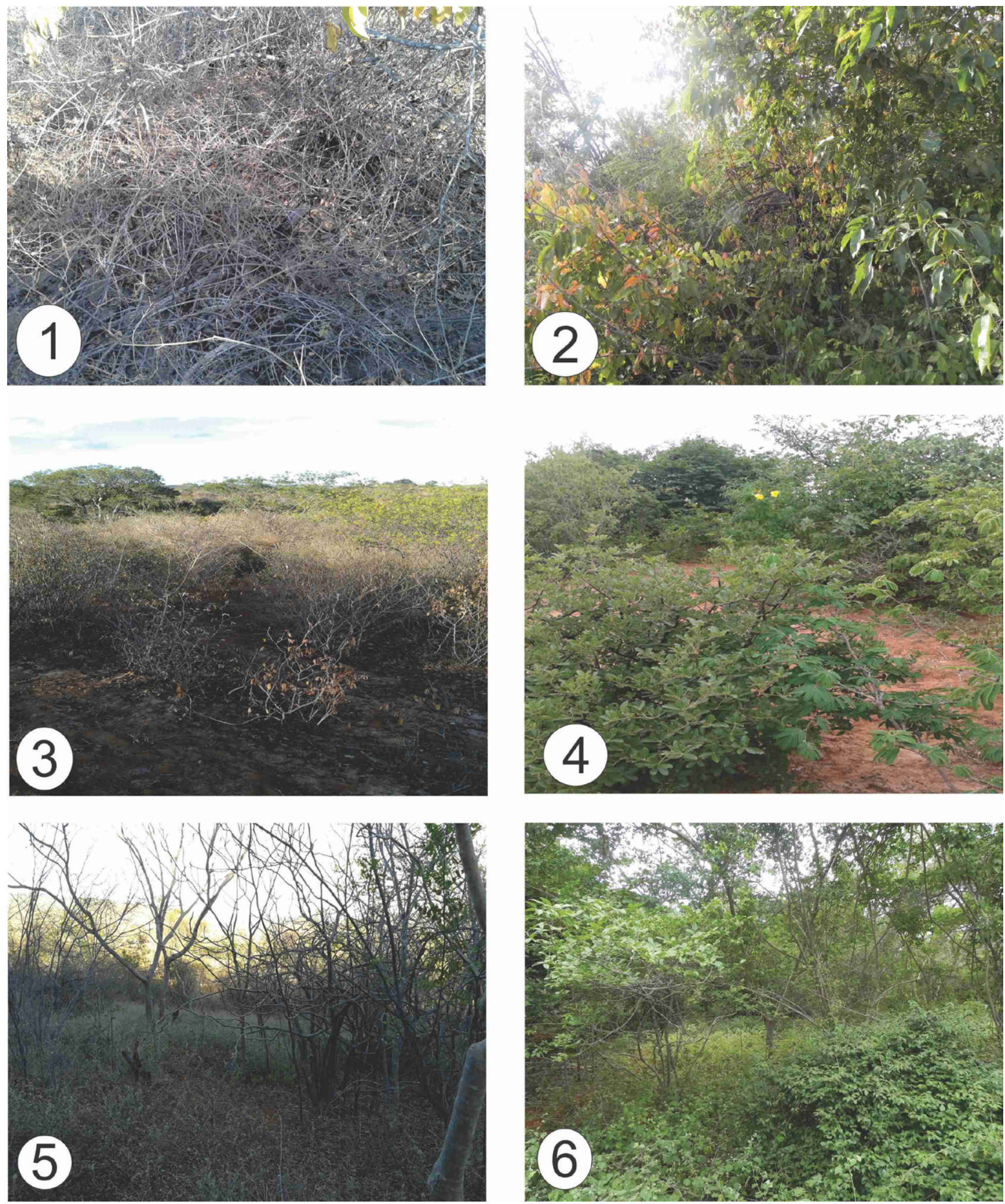

Figs 1-6. Áreas de Caatinga amostradas no município de Ibiassucê, Bahia, Brasil no período seco (PS) e chuvoso (PC). Morro da Tapera: PS (Fig. 1), PC (Fig. 2); Quilombo Santo Inácio: PS (Fig. 3), PC (Fig. 4); Fazenda Tanque da Mandioca: PS (Fig. 5), PC (Fig. 6).

morfotipos de galhas e suas plantas hospedeiras já registradas em ambientes de Caatinga, em virtude das galhas estarem vazias ou com apenas as fases imaturas. O material está depositado na Coleção de Entomologia do Museu Nacional/ UFRJ (MNRJ).
As plantas hospedeiras foram coletadas, herborizadas e aquelas com partes reprodutivas foram depositadas no Herbário HUNEB - Coleção Caetité. A identificação do material botânico foi realizada principalmente com auxílio de chaves analíticas encontradas em literatura especializada e 
também por comparação com material depositado no HUNEB e no Herbário da Universidade Estadual de Feira de Santana (HUEFS). A listagem dos táxons está organizada em ordem alfabética por família, gênero e espécie, de acordo com APG IV (2016).

A riqueza de galhas foi comparada entre os três estágios de conservação dos ambientes e as estações (chuvosa e seca) aplicando um delineamento fatorial duplo ( $3 \times 2)$. As médias foram comparadas após análise de variância, pelo teste de Tukey a 5\% de probabilidade, para isso utilizou-se o software estatístico SISVAR 5.6 (FERREIRA, 2014).

\section{RESULTADOS}

Foram registrados 20 morfotipos de galhas distribuídos em nove espécies de plantas, pertencentes a oito famílias botânicas (Tab. II). Do total de galhas, nove foram observadas no período seco, 11 no chuvoso e nove em ambas as estações (Figs 7-26; Tab. II).

Nas áreas intermediárias foi registrada a maior riqueza, oito morfotipos em sete espécies vegetais, sendo que do total de morfotipos encontrados, seis foram exclusivos dessas áreas. Nas áreas antropizadas, sete espécies hospedaram sete morfotipos de galhas, com cinco destes registrados unicamente nesses ambientes. Nas áreas conservadas encontraram-se cinco morfotipos distribuídos em quatro espécies de plantas hospedeiras, dos quais quatro deles ocorreram exclusivamente nesses ambientes. Apesar dessas diferenças, a riqueza não foi influenciada pelo grau de conservação das áreas estudadas no período de avaliação (seco e chuvoso), segundo análise de variância e teste de Tukey a $5 \%$ de probabilidade (Tab. III).

$\mathrm{O}$ maior número de galhas foi encontrado em arbustos $(n=5)$, em comparação com as demais formas de vida, erva ( $n=3)$, trepadeira (3), subarbusto (2) e arbóreo $(\mathrm{n}=1)$. As famílias de plantas com maior riqueza foram: Bignoniaceae, Leguminosae e Sapindaceae, com oito, quatro e dois morfotipos, respectivamente. Adenocalymma Mart. ex Meisn. (Bignoniaceae) ( $\mathrm{n}=7$ ), Mimosa L. ( $\mathrm{n}=3$ ) e Senegalia Raf. ( $\mathrm{n}=2$ ) (Leguminosae-Caesalpinoideae) foram os gêneros com maior riqueza de galhadores (Tab. II). Mimosa tenuiflora (Willd.) Poir. foi a espécie que apresentou maior número de morfotipos $(n=3)$. O órgão mais galhado foi a folha $(60 \%)$, com ocorrências tanto na face adaxial quanto na abaxial, seguida do caule (40\%). Não foram observadas galhas em flores e frutos, mesmo estes sendo vistoriados. No que diz respeito à forma da galha, a maioria foi globoide $(37,1 \%)$, seguidas de fusiforme $(31,4 \%)$ e lenticular $(14,2 \%)$, com coloração marrom $(57,1 \%)$ e verde $(42,9 \%)$, glabras $(70 \%)$, ocorrendo agrupadas (55\%) e apresentando apenas uma câmara larval $(82,9 \%)$ (Tab. II).

Foram identificados galhadores pertencentes às ordens Coleoptera (Curculionidae), Diptera (Cecidomyiidae) e Lepidoptera. Dípteros induziram galhas em Croton echioides Baill. (Euphorbiaceae) e M. tenuiflora; Coleoptera em Senegalia paganuccii Seigler, Ebinger \& P.G. Ribeiro e M. tenuiflora. Indivíduos de Lepidoptera induziram galhas em Neocalyptrocalyx longifolium (Mart.) Cornejo \& Iltis (Capparaceae), M. tenuiflora e Calliandra macrocalyx Harms (Leguminosae-Caesalpinoideae). A fauna associada às galhas foi composta por espécies das ordens Hemiptera, Hymenoptera e Psocoptera. Além desses, foi observado uma espécie de Araneae sucessora em galhas foliares abandonadas pelo indutor em Adenocalymma sp.

\section{DISCUSSÃO}

A fauna de insetos galhadores encontradas nas áreas de Caatinga de Ibiassucê é menor que a de outras da Região Nordeste (SANTOS et al., 2011; CARVALHO-Fernandes et al., 2012). Estudos recentes sobre insetos galhadores realizados em ambientes de Caatinga, com diferentes esforços de coleta, registraram 33 morfotipos de galhas no Parque Nacional do Vale do Catimbau, Buíque, Pernambuco (Santos et al., 2011) e 25 morfotipos em diferentes áreas próximas à Usina Hidroelétrica de Xingó (CHESF) (CARvalho-Fernandes et al., 2012).

Embora a riqueza de galhas tenha sido maior nas áreas intermediárias quando comparadas às áreas preservadas e antropizadas, não houve diferença significativa entre elas, não corroborando a hipótese aqui testada de que haveria um maior número de galhas em ambientes antropizados. O único estudo que observou a distribuição de insetos galhadores em áreas de caatinga em seus diferentes níveis de conservação, realizado com metodologia similar, constatou uma riqueza maior de morfotipos de galhas nas áreas preservadas do que nas áreas intermediárias e antropizadas (CARVALHO-FERNANDES et al., 2012), diferentemente do que foi encontrado no presente estudo.

Os insetos galhadores podem ser úteis na avaliação do impacto humano (FERNANDES \& MARTINS, 1985; FERNANDES et al., 1995, 2009; Julião et al., 2005; FeRnANDES \& NeGReIRos, 2006) por estabelecer uma interação espécieespecífica de fácil identificação através dos seus morfotipos e por serem sensíveis às mudanças em suas plantas hospedeiras ou em seu ambiente (Toмa et al., 2014). Algumas evidências apontam que esses insetos podem ocorrer em grande abundância em habitats modificados pela ação antrópica (Altamirano et al., 2016), porém alguns galhadores são mais sensíveis às pequenas alterações ambientais, ocorrendo apenas em ambientes conservados, o que os torna melhores indicadores da qualidade ambiental (Julĩ̃o et al., 2005). Os resultados obtidos neste estudo não corroboram nenhuma das evidências. Apesar disso, entendemos que há necessidade da continuidade de estudos sobre a diversidade de insetos galhadores e suas respostas às mudanças ambientais em escalas de tempo mais longas e abrangendo áreas mais extensas, principalmente porque os estudos que mostram o efeito da antropização sobre a comunidade de galhadores na Caatinga são relativamente recentes.

A maioria das galhas foi encontrada no estrato arbustivo, seguido pelo herbáceo, subarbustivo e arbóreo, coincidindo com os dados da literatura (GONÇALVES-ALVIM \& FERNANDES, 2001; FAGUNDES et al., 2001). Este fato pode 


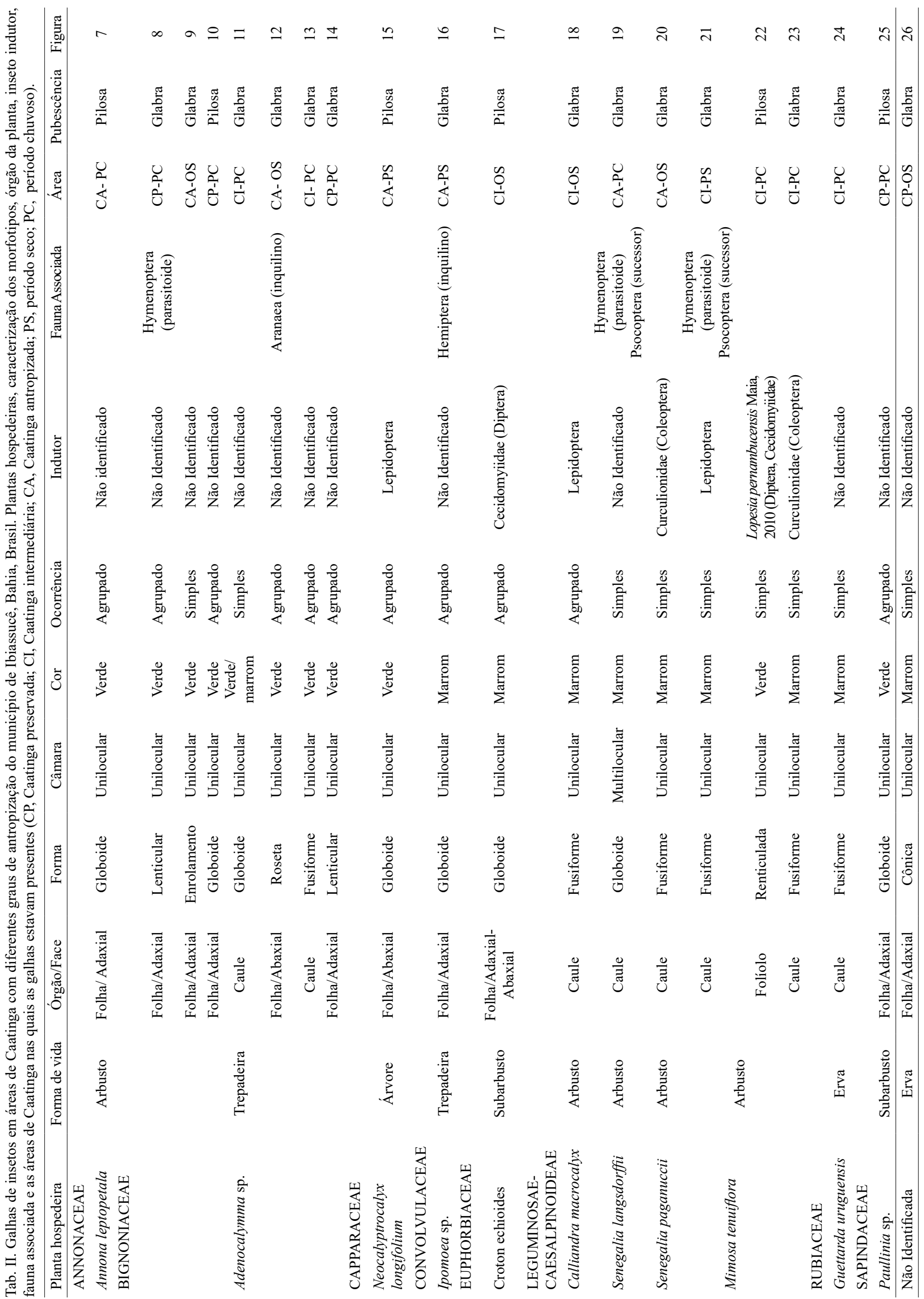


Tab. III. Comparação sazonal da riqueza de galhas em áreas de Caatinga com diferentes graus de antropização do município de Ibiassucê, Bahia, Brasil (*Médias seguidas da mesma letra minúscula na coluna e maiúscula na linha, não diferem entre si pelo teste de Tukey a 5\% de probabilidade).

\begin{tabular}{lcc}
\hline \multirow{2}{*}{ Áreas de estudo } & \multicolumn{2}{c}{ Número de espécies hospedeiras } \\
\cline { 2 - 3 } & Chuvoso & $1,25 \mathrm{aA}$ \\
Antropizada & $0,5 \mathrm{aA} *$ & $0,25 \mathrm{aA}$ \\
Preservada & $0,75 \mathrm{aA}$ & $0,75 \mathrm{aA}$ \\
\cline { 2 - 3 } Intermediária & $1 \mathrm{aA}$ & Seco \\
& & Número de morfotipos de galhas \\
\cline { 2 - 3 } & Chuvoso & $1,25 \mathrm{aA}$ \\
Antropizada & $0,5 \mathrm{aA}$ & $0,25 \mathrm{aA}$ \\
\hline Preservada & $1 \mathrm{aA}$ & $0,75 \mathrm{aA}$ \\
\hline
\end{tabular}

estar relacionado à complexidade estrutural das plantas. Plantas estruturalmente mais complexas apresentando uma maior variação química e anatômica oferecem um maior número de microhábitats para insetos herbívoros (FERNANDES \& PRICE, 1988).

No Brasil, inventários de diversidade de galhas em áreas de Caatinga (CARVAlHo-Fernandes et al., 2012; AlCÂNTARA et al., 2017) apontam Leguminosae e Bignoniaceae como as principais famílias hospedeiras de galhas, similar ao presente estudo. Essas famílias possuem grande representatividade na composição florística em ambientes de Caatinga (SAMPAIO, 1995, 2002; Rodal et al., 1999; QUEIROZ, 2009), corroborando, desta forma, com a hipótese do tamanho do táxon na qual famílias com maior número de espécies vegetais possuem maior riqueza de morfotipos de galhas (Fernandes \& PRICE, 1992).

$\mathrm{O}$ maior número de morfotipos foi encontrado em indivíduos pertencentes a Adenocalymma, Senegalia e Mimosa. Estes gêneros são encontrados frequentemente na composição vegetal da Caatinga (GiulietTi et al., 2004). Além disso, Senegalia e Mimosa apresentaram elevada riqueza de galhadores em estudos realizados no domínio fitogeográfico da Caatinga (NogueIRA et al., 2016), enquanto que Adenocalymma é citado pela primeira vez como gênero hospedeiro de galhas em ambientes de Caatinga.

A indução preferencial em folhas corrobora o padrão mundial observado por Felt (1940) e também em levantamentos realizados em diversos ecossistemas no Brasil, com diferentes métodos (e.g., JuLIão et al., 2002; Oliveira \& Maia, 2005; Fernandes et al., 2009; Maia, 2013; NogueIRA et al., 2016). Padrão semelhante vem sendo observado nos estudos já realizados na Caatinga (SANTOS et al., 2011; CARVAlHo-Fernandes et al., 2012; AlCÂNTARA et al., 2017).

Galhas globoides, fusiformes e lenticulares foram as mais frequentes, padrão equivalente foi observado tanto em outras áreas da mesma vegetação (CARVALHO-FERNANDES et al., 2012; AlCÂNTARA et al., 2017) quanto em diferentes ecossistemas (e.g., MAIA, 2013; COSTA et al., 2014a,b). Nas áreas estudadas, $43 \%$ das galhas apresentaram tricomas na sua superfície externa. Apesar das galhas com tricomas não serem a maioria, esse valor é alto quando comparado a estudos de diversidade de galhas realizados em diferentes ecossistemas brasileiros (ARAúJO et al., 2007; CARVALHO-FERNANDES et al., 2012; NogueIRA et al., 2016). Em regiões semiáridas, como a Caatinga, os tricomas são importantes estruturas que funcionam como uma proteção contra dessecação e ação de predadores e parasitas (APPEZZATO-DA-GLÓRIA \& CARMEllo-Guerreiro, 2006). Dessa forma, em galhas, os tricomas podem conferir um microclima adequado para o desenvolvimento do inseto (WeSTPHAL, 1992; BoczeK \& GRIFFITHS, 1994), protegendo-o contra as altas temperaturas e estresse hídrico.

A maioria das galhas apresentou apenas uma câmara larval e distribuiu-se de maneira agrupada. Este resultado corrobora o padrão encontrado em estudos de diversidade de galhas em diferentes fitofisionomias no Brasil, tais como Cerrado (Gonçalves-Alvim \& Fernandes, 2001) e Mata Atlântica (FERNANDES et al., 2009). Quanto à coloração dos morfotipos, a maioria das galhas apresentou-se verde e marrom, e com a mesma coloração do órgão vegetal hospedeiro, estando de acordo com a literatura (SANTOS et al., 2010). No entanto, algumas galhas foliares apresentaram coloração amarelas e vermelhas; possivelmente essa característica deve-se à degradação das clorofilas e síntese de carotenoides e antocianinas nos tecidos (CASTRO et al., 2012).

Os insetos que têm o hábito de induzir galhas pertencem às ordens Coleoptera, Diptera, Hemiptera, Hymenoptera, Lepidoptera e Thysanoptera (MAIA, 2013). Três ordens de insetos indutores de galhas puderam ser identificadas neste estudo. Uma grande proporção das galhas estava vazia ou com apenas as fases imaturas, o que dificultou a identificação dos indutores. Na região Neotropical, Diptera é a principal ordem de insetos galhadores podendo induzir galhas em todos os órgãos vegetais (MAIA \& AzEvEDo, 2009). Em contrapartida, espécies de Lepidoptera comumente induzem galhas em caules (MAIA, 2006), similar ao encontrado neste estudo na planta hospedeira Mimosa tenuiflora.

Além dos indutores podem ser encontrados no interior das galhas outros organismos, atuando como inquilinos, predadores, sucessores e parasitoides. De acordo com MaIA (2001), Pseudoscorpiones, Hymenoptera, Coleoptera, Lepidoptera e Diptera fazem parte dessa fauna associada, sendo os Hymenoptera o grupo mais frequente, a maioria parasitoide, e alguns fitófagos (MAIA \& AZEVEDO, 2009). A fauna de artrópodes associada às galhas nas áreas estudadas inclui Araneae, Hemiptera, Hymenoptera e Psocoptera. Os 

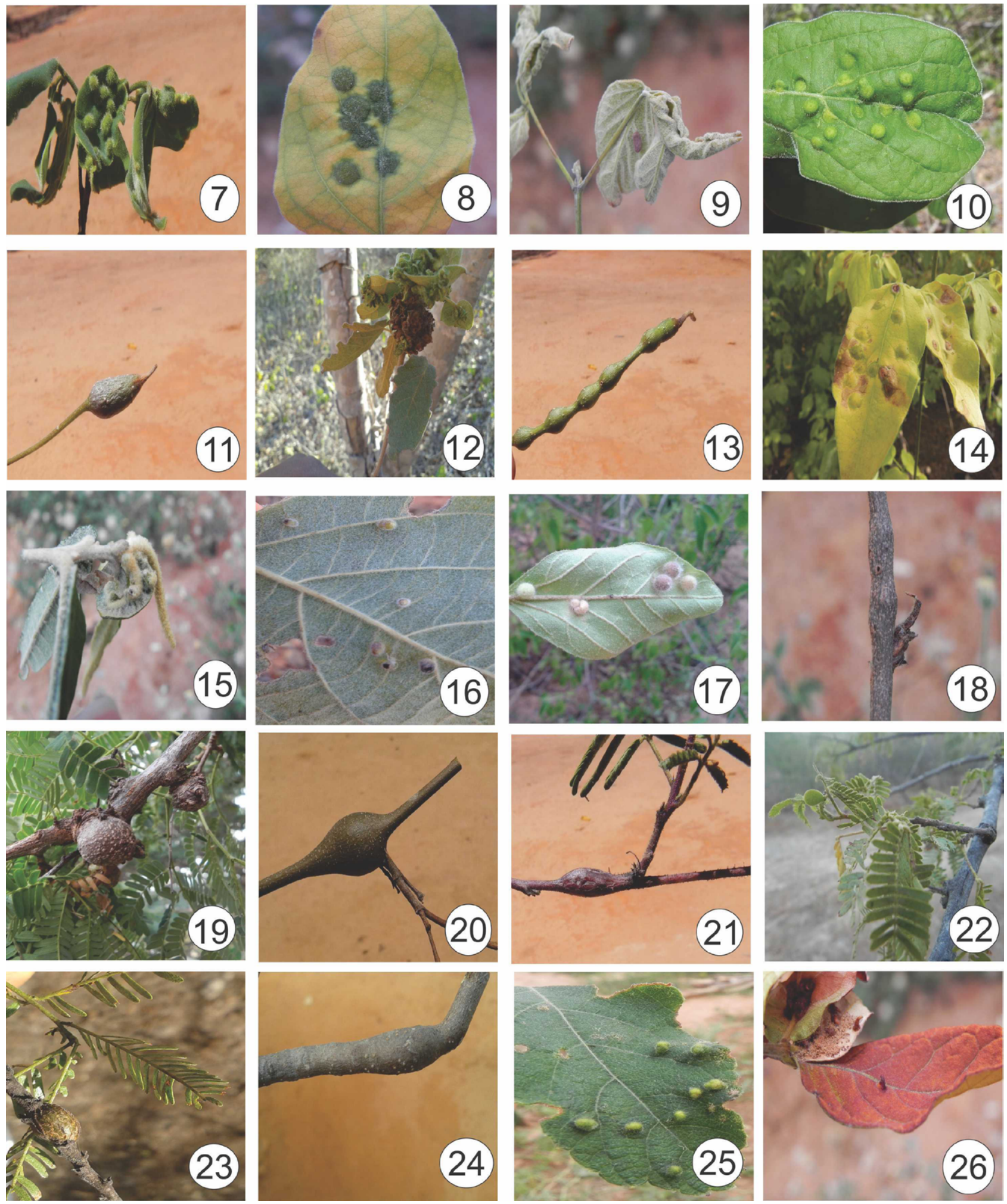

Figs 7-26. Diversidade de galhas entomógenas em áreas de Caatinga do município de Ibiassucê, Bahia, Brasil: 7, Annona leptopetala (R.E.Fr.) H. Rainer; 8-14, Adenocalymma. sp.; 15, Neocalyptrocalyx longifolium (Mart.) Cornejo \& 1ltis; 16, Ipomoea sp.; 17, Croton echioides Baill.; 18, Calliandra macrocalyx Harms; 19, Senegalia langsdorffii (Benth.) Seigler \& Ebinger; 20, Senegalia paganuccii Seigler, Ebinger \& P.G. Ribeiro; 21-23, Mimosa tenuiflora (Willd.) Poir.; 24, Guettarda uruguensis Cham. \& Schltdl; 25, Paullinia sp.; 26, Indeterminada.

inquilinos ocorreram em apenas um morfotipo, representado por Hemiptera. Foram encontrados Hymenoptera parasitoides associados a três morfotipos de galhas. Com relação aos sucessores, a fauna foi composta por Psocoptera e Araneae.
Em outras áreas de Caatinga essas ordens também já foram registradas com os mesmos hábitos verificados nesse estudo (SANtos et al., 2011; CARVAlHo-Fernandes et al., 2012), com exceção de Psocoptera que está sendo registrado pela primeira 
vez aqui compondo a fauna de invertebrados associada às galhas de insetos em ambientes de Caatinga.

Todos os morfotipos encontrados nos diferentes estágios de sucessão ecológica em áreas de Caatinga são novos para Ibiassucê. Além disso, registra-se pela primeira vez a ocorrência de galhas em Neocalyptrocalyx longifolium (Mart.) Cornejo \& Iltis e Guettarda uruguensis Cham. \& Schltdl. Esses resultados reforçam a importância desse tipo estudo e evidenciam a necessidade da realização de inventários de galhas em áreas pouco ou não amostradas da Caatinga.

Agradecimentos. À Fundação de Amparo à Pesquisa do Estado da Bahia (FAPESB) pela concessão da bolsa de iniciação científica à primeira autora através do Programa Institucional de Bolsa de Iniciação Científica (PIBIC/UNEB). Ao CNPq (Proc. n 406111/2016-2) e à FAPESB (Proc. ${ }^{\circ}$ 9648/2015) pelo apoio financeiro ao projeto. À FAPERJ pela bolsa a S.P. Carvalho-Fernandes (Proc. E-26/203.170/2015). Nossos agradecimentos também aos pesquisadores da Universidade Estadual de Feira de Santana pela ajuda na identificação do material botânico e a Profa. Dra. Francyane Braga (UNEB) pela ajuda com as análises estatísticas.

\section{REFERÊNCIAS BIBLIOGRÁFICAS}

Alcântara, J. A.; Souza, E. B. \& Braga, P. E. T. 2017. Ocorrência e caracterização de galhas em duas áreas do noroeste do Ceará, Brasil. Natureza on line 15:33-40.

Almeida-Neto, M.; Prado, P. I. \& Lewinsohn, T. M. 2011. Phytophagous insect fauna tracks host plant responses to exotic grass invasion. Oecologia 165:1051-1062.

Altamirano, A.; Valladares, G.; Kuzmanich, N. \& Salvo, A. 2016. Galling insect in a fragmented forest: incidence of habitat loss, edge effects and plant availability. Journal of Insect Conservation 20:119127

APG IV. 2016. An update of the Angiosperm Phylogeny Group classification for the orders and families of flowering plants: APG IV. Botanical Journal of the Linnean Society 181:1-20.

ApPeZzato-DA-Glória, B. \& CARMELlo-Guerreiro, S. M. 2006. Anatomia vegetal. 2ed. Viçosa, Universidade Federal de Viçosa. 438p.

Araújo, W. S.; Gomes-Klein, V. L. \& Santos, B. B. 2007. Galhas entomógenas associadas à vegetação do Parque Estadual da Serra dos Pireneus, Pirenópolis, Goiás, Brasil. Revista Brasileira de Biociências 5(1):45-47.

AraúJo, W. S.; Porfírio-Júnior, E. D.; Jorge, V. A. \& Espírito-Santo FiLHO, K. 2012. Plantas hospedeiras e galhas entomógenas em subbosques de florestas tropicais do Pará, Brasil. Insula 41:59-72.

Araújo, W. S.; Espírito-Santo, K.; Bergamini, L. L.; Gomes, E. \& Morato, S. A. A. 2014. Plant Habitat conversion and galling insect richness in tropical rainforest under mining effects. Journal of Insect Conservation 18:1147-1152

BoczeK, J. D. \& GRIFFTHS, D. 1994. Structure and systematics of eriophyid mites (Acari: Eriophyoidea) and their relationship to host plants. $I n$ : Williams, M. A. J. ed. Plant galls: organisms, interactions, populations. New York, Clarendon Press, p. 119-129.

Bolger, D. T.; Suarez, A. V.; Crooks, K. R.; Morrison, S. A. \& Case, T. J. 2000. Arthropods in urban habitat fragments in Southern California: area, age, and edge effects. Ecological Applications 10:1230-1248

Brasileiro, R. S. 2009. Alternativas de desenvolvimento sustentável no semiárido nordestino: da degradação à conservação. Scientia Plena 5(5):1-12.

CAR - Companhia de Desenvolvimento e AÇão Regional - BA. 2007. Programa de Desenvolvimento Regional Sustentável: PDRS Serra Geral. Salvador, Secretaria de Planejamento, Ciência e Tecnologia, 338p.

Carvalho-Fernandes, S. P.; Almeida-Cortez, J. S. \& Ferreira, A. L. N. 2012. Riqueza de galhas entomógenas em áreas antropizadas e preservadas de Caatinga. Revista Árvore 32(2):269-277.
Castro, A. C.; Oliveira, D. C.; Moreira, A. S. F. P.; Lemos-Filho, J. P. \& ISAIAS, R. M. S. 2012. Source-sink relationship and photosynthesis in the horn-shaped gall and its host plant Copaifera langsdorffii Desf. (Fabaceae). South African Journal of Botany 83:121-126.

Christie, F. J.; Gerasimos, C. \& Hochuli, D. F. 2010. Urbanization affects the trophic structure of arboreal arthropod communities. Urban Ecosystems 13:169-180.

Costa, E. C.; Carvalho-Fernandes, S. P. \& Santos-Silva, J. 2014a. Galhas de insetos em uma área de transição Caatinga-Cerrado no Nordeste do Brasil. Sitientibus, Série Ciências Biológicas 14:1-9.

Costa, E. C.; Carvalho-Fernandes, S. P. \& SAntos-Silva, J. 2014b. Galhas entomógenas associadas à Leguminosae do entorno do riacho Jatobá, Caetité, Bahia, Brasil. Revista Brasileira de Biociências 12(2):115-120.

Embrapa SOLOS. 2006. Solos do Nordeste. UEP, Recife. Disponível em $<$ http://www.uep.cnps.embrapa.br/solos/>. Acesso em 28 dez. 2016.

Fagundes, M.; Farias, M. L. \& Fernandes, G. W. 2001. Efeitos da distribuição de Baccharis dracunculifolia (Asteraceae) na abundância e no parasitismo de galhas de Neopelma baccharidis (Homoptera: Psyllidae). Unimontes Científica 1(1):97-103.

Felt, E. P., 1940. Plant galls and gall makers. Ithaca, Comstock Publishing. $364 \mathrm{p}$.

Fernandes, G. W. A. \& Martins, R. P. 1985. Tumores de plantas: As galhas. Ciência Hoje 4:58-64.

Fernandes, G. W. A. \& Negreiros, D. 2006. A comunidade de insetos galhadores da RPPN Fazenda Bulcão, Aimorés, Minas Gerais, Brasil. Lundiana 7:111-120.

Fernandes, G. W. \& Price, P. W. 1988. Biogeographical gradients in galling species richness: tests of hypotheses. Oecologia 76:161-167.

Fernandes, G. W. \& Price, P. W. 1992. The adaptive significance of insect gall distribution: survivorship of species in xeric and mesic habitats. Oecologia 90:14-20.

Fernandes, G. W. A.; Paula, A. S. \& Loyola JR., R. 1995. Distribuição diferencial de insetos galhadores entre habitats e seu possível uso como indicadores. Vida Silvestre Neotropical 4:133-139.

Fernandes, S. P. C.; Castelo-Branco, B. P.; Albuquerque, F. A.; Ferreira, A. L. N.; Brito-Ramos, A. B.; Braga, D. V. V. \& AlmeidaCortez, J. 2009. Galhas entomógenas em um fragmento urbano de Mata Atlântica no centro de endemismo de Pernambuco. Revista Brasileira de Biociências 7(3):240-244.

FERREIRA, D. F. 2014. Sisvar: a Guide for its Bootstrap procedures in multiple comparisons. Ciência e Agrotecnologia 38(2):109-112.

Giulietti, A. M.; Bocage Neta, A. L.; Castro, A. A. J. F.; GamarraRojas, C. F. L.; Sampaio, E. V. S. B.; Virgínio, J. F. \& Harley, R. M. 2004. Diagnóstico da vegetação nativa do bioma Caatinga. In: Silva, J. M. C.; Tabarelli M.; Fonseca, M. T. \& Lins, L. V. orgs. Biodiversidade da Caatinga: áreas e ações prioritárias para a conservação. Brasília, JMMA, UFPE, Conservation International do Brasil, Fundação Biodiversitas, Embrapa Semi-Árido, p.48-90.

GonÇALVES-AlVim, S. J. \& FernANDES, G. W. 2001. Biodiversity of galling insects: historical, community and habitat effects in four neotropical savannas. Biodiversity and Conservation 10:79-98.

González, E.; Salvo, A. \& Valladares, G. 2015. Arthropods on plants in a fragmented neotropical dry forest: a functional analysis of area loss and edge effects. Insect Science 22:129-138.

GonZÁlez, E.; Salvo, A. \& VAlLadares, G. 2017. Natural vegetation cover in the landscape and edge effects: differential responses of insect orders in a fragmented forest. Insect Science 24:891-901.

Hines, J.; Lynch, M. E. \& DenNo, R. F. 2005. Sap-feeding insect communities as indicators of habitat fragmentation and nutrient subsidies. Journal of Insect Conservation 9:261-280.

IBGE - Instituto Brasileiro de Geografia e Estatística. 2006. Censo agropecuário 2006: Brasil, grandes regiões e unidades da federação. Rio de Janeiro, IBGE. 146p.

IBGE - Instituto Brasileiro de Geografia e Estatística. 2017. Ibiassucê. Disponível em $<$ https://cidades.ibge.gov.br/painel/historico.php?lang $=\&$ codmun $=291200 \&$ search=bahia $\mid$ ibiassuce $\mid$ infograficos:-historico $>$. Acesso em 28 Nov. 2017.

Isaias, R. M. S.; Carneiro, R. G. S.; Oliveira, D. C. \& Santos, J. C. 2013. Illustrated and annotated checklist of Brazilian gall morphotypes. Neotropical Entomology 42(3):230-239. 
Julião, G. R.; Amaral, M. E. \& Fernandes, G. W. 2002. Galhas de insetos e suas plantas hospedeiras no Pantanal Sul-Mato-Grossense. Naturalia 27:47-74.

Julião, G. R.; Fernandes, G. W.; Negreiros, D.; Bedê, L. \& Araúuo, R. A. 2005. Insetos galhadores associados a duas espécies de plantas invasoras de áreas urbanas e peri-urbanas. Revista Brasileira de Entomologia 49(1):97-106.

KAARTINEN, R. \& RosLin, T. 2011. Shrinking by numbers: landscape context affects the species composition but not the quantitative structure of local food webs. Journal of Animal Ecology 80:622-631.

MAIA, V. C. 2001. The gall midges (Diptera, Cecidomyiidae) from three restingas of Rio de Janeiro State, Brazil. Revista Brasileira de Zoologia 18(2):583-629.

MaIA, V. C. 2006. Galls of Hemiptera, Lepidoptera and Thysanoptera from Central and South America. Publicações Avulsas do Museu Nacional 110:3-22.

MAIA, V. C. 2013. Insect galls from restingas of Southeastern Brazil, with new records. Biota Neotropica 13(1):184-209.

Maia, V. C. \& Azevedo, M. A. P. 2009. Micro-himenópteros associados com galhas de Cecidomyiidae (Diptera) em Restingas do Estado do Rio de Janeiro (Brasil). Biota Neotropica 9:151-164.

MDA - MinistéRIO DO DESENVOLVIMENTO AGRÁRIO. 2010. Plano territorial de desenvolvimento rural sustentável do sertão produtivo. Caetité, MDA. $121 \mathrm{p}$.

Moreira, R. G.; Fernandes, G. W.; Almada, E. D. \& Santos, J. C. 2007. Galling insects as bioindicators of land restoration in an area of Brazilian Atlantic Forest. Lundiana 8(2):107-112.

Nogueira, R. M.; Costa, E. C.; Carvalho-Fernandes, S. P. \& SantosSILVA, J. 2016. Insect galls from Serra Geral, Caetité, BA, Brazil. Biota Neotropica 16(1):1-10.

Oliveira, J. C. \& MAIA, V. C. 2005. Ocorrência e caracterização de galhas de insetos na restinga de Grumari (Rio de Janeiro, RJ, Brasil). Arquivo do Museu Nacional 63:669-675.

Queiroz, L. P. 2009. Leguminosas da Caatinga. Feira de Santana, Universidade Estadual de Feira de Santana. 467p.

Rodal, M. J. N.; Nascimento, L. M. \& Melo, A. L. 1999. Composição florística de um trecho de vegetação arbustiva caducifólia, no município de Ibimirim, Pernambuco, Brasil. Acta Botanica Brasilica 13(1):15-28.

SAmpaio, E. V. S. B. 1995. Overview of the Brazilian caatinga. In: Bullock, S. H.; Mooney, H. A. \& Medina, E. eds. Seasonal dry tropical forests. Cambridge, Cambridge University Press, p. 35-63.

Sampaio, E. V. S. B. 2002. Uso das plantas da Caatinga. In: Sampaio, E. V. S. B.; Giulietti, A. M.; Virgínio, J. \& Gamarra-Rojas, C. F. L. eds. Vegetação e Flora da Caatinga. Recife, Associação Plantas do Nordeste (APNE) e Centro Nordestino de Informação Sobre Plantas, p.49-90.

Santos, B. B.; Ferreira, H. D. \& Araújo, W. S. 2010. Ocorrência e caracterização de galhas entomógenas em uma área de floresta estacional semidecídua em Goiânia, Goiás, Brasil. Acta Botanica Brasilica 24(1):243-249.

Santos, J. C.; Almeida-Cortez, J. S. \& Fernandes, G. W. 2011. Richness of gall-inducing insects in the tropical dry forest (Caatinga) of Pernambuco. Revista Brasileira de Entomologia 55(1):45-54.

TABARELli, M. 1998. Reserva Ecológica de Dois Irmãos: estudos em um remanescente de Mata Atlântica em área urbana. In: Machado, I. C.; Lopes A. V. \& PôRTo, K. C. orgs. Reserva Ecológica de Dois Irmãos: estudos em um remanescente de Mata Atlântica em área urbana (Recife, Pernambuco, Brasil). Recife, Editora da Universidade Federal de Pernambuco, p. 311-323.

TABARELli, M. \& Silva, J. M. C. 2002. Áreas prioritárias para a conservação, utilização sustentável e repartição de benefícios da biodiversidade do bioma Caatinga. In: Araúso, E. L.; Moura, A. N.; Sampaio, E. V. S. B.; Gestinari L. M. S. \& Carneiro, J. M. T. eds. Biodiversidade, conservação e uso sustentável da flora do Brasil. Recife, Universidade Federal Rural de Pernambuco, Imprensa Universitária. 298p.

Toma, T. S.; Fernandes, G. W.; De Souza, D. G.; Tabarelli, M. \& SANTOS, J. C. 2014. Galling insects as indicators of habitat quality. In: Fernandes, G. W. \& Santos, J. C. eds. Neotropical insect galls. Netherlands, Springer, p. 143-150.

Westrhal, E. 1992. Cecidogenesis and resistance phenomena in miteinduced galls. In: SHORTHOUSE, J. D. \& ROHFritsch, O. eds. Biology of insect-induced galls. New York, Oxford University Press, p.157-170. 